\title{
ELMAPA DELMUNDOROMANO
}

\author{
Adela Cepas Palanca*, \\ Javier Sánchez-Palencia, \\ Domingo Plácido**
}

RESUMEN: Este trabajo es una revisión de la historia del proyecto de Tabula Imperii Romano desde sus inicios en 1928. Se expone también la activa colaboración de España y las distintas fases por las que ha atravesado en este país la elaboración del Mapa del Mundo Romano.

ABSTRACT: This paper is a review of the history of the project "Tabula Imperii Romani" since is started in 1928. It is also explained the active collaboration of Spain and the diferent phases that the elaboration of the Map of the Roman World has gone througth in this country.

\section{O.G.S. CRaWford y la Tabula ImPeriI Romani}

Hablar de la Tabula Imperii Romani, denominación internacional de lo que en un principio se llamó simplemente "Mapa del Mundo Romano", exige hacer una breve referencia a O.G.S. Crawford (1886-1957). Aunque pueda parecer paradójico, la idea de elaborar un mapa del Imperio Romano no surge de un historiador de la Antigüedad, ni de un arqueólogo, sino de un geógrafo cuyo interés científico basculó siempre entre la arqueología y la geografía. Aunque su contacto con la Antigüedad empezó en sus años escolares, se graduó en Geografía en la universidad de Oxford, materia en la que inició su vida profe-

* C.E.H. del C.S.I.C.

*** Universidad Complutense. 
sional como profesor de la Escuela de Geografía de esta ciudad ${ }^{1}$. Durante la Primera Guerra Mundial sirvió en el ejército de tierra y en las fuerzas aéreas y en 1920 el Servicio Cartográfico inglés (Ordnance Survey) creó el puesto de Funcionario Arqueológo para el que fue elegido y en el que permaneció hasta su jubilación en 1946. Fue fundador y editor de Antiquity, revista en la que trabajó desde 1927 hasta poco antes de su muerte en 1957.

Así pues, Crawford entra en el mundo de la arqueología y de la historia con una sólida formación geográfica y cartográfica, hecho que marcará toda su obra al aplicar a aquellas materias la metodología aprendida durante su años universitarios, en concreto la geografía humana, disciplina que en ese momento estaba enfocada hacia el análisis del medio geográfico y ambiental de las modernas comunidades subdesarrolladas. Desde un principio se aleja del enfoque tradicional de la historia, materia a la que nunca le interesó acercarse con las armas tradicionales del historiador ${ }^{2}$.Tampoco era un arqueólogo de campo, ya que su actividad científica se centró en lo que él denominó Field Archaeology, es decir, en la prospección, actividad que requería tanto un buen conocimiento de topografía como la necesaria habilidad cartográfica para plasmar en un mapa los datos recogidos sobre el terreno. Fue, sin duda, uno de los pioneros de la fotointerpretación aplicada a la arqueología, técnica aprendida en sus años de servicio en el ejército como observador aéreo durante la Primera Guerra Mundial.

Sus planteamientos teóricos se basaban en un principio muy simple: los datos históricos y arqueológicos sólo pueden entenderse plenamente si se localizan con la mayor exactitud posible en el tiempo y en el espacio.Así como el aspecto temporal requiere la elaboración de un sistema cronológico, el espacial necesita un soporte cartógrafico adecuado, ya que todo lugar, antiguo o moderno, cobra significado en el medio que lo rodea. En este sentido son reveladoras sus palabras, the archaeologist or bistorian who bas not disciplined bis thought so that be thinks in terms of these two fundamental dimensions will never really understand bis subject ${ }^{3}$. Por tanto, se le puede considerar un adelantado en el uso de buena parte de las técnicas espaciales que han alcanzado auge en las dos últimas décadas.

Este concepto de la arqueología es el que domina en los múltiples proyectos que llevó a cabo en el Ordnance Survey. El primero fue la elaboración de un mapa de su país en época romana, publicado por primera vez en 1924, al que pronto siguió una segunda edición en 1934 y una tercera en 1951. La experiencia adquirida le servió de gran utilidad y supuso un importante punto de partida para trabajos posteriores de mayor envergadura como su gran proyec-

\footnotetext{
1. KIRWAN, L.P., 1958: 14.

2. CRAWFORD, O.G.S., 1949: 128 ss.

3. CRAWFORD, O.G.S., 1953: 40 ss.
} 
to internacional de cartografía: el Mapa del Mundo Romano a escala 1: 1.000.000. Fue un precursor de la arquelogía del paisaje como muestran sus trabajos sobre la arqueología del condado de Wessex, desde la prehistoria hasta el período napoleónico, y los mapas secuenciales ${ }^{4}$ que constituyen su segundo proyecto y una de sus contribuciones más originales al Ordnance Survey ${ }^{5}$.

No parecen justas las críticas que recibió por su peculiar visión de la Arqueología, en las que predominaba la acusación de localismo ${ }^{6}$, cuando bien se sabe que su principal proyecto fue una obra tan universal como la elaboración del mapa del Mundo Romano.

Este proyecto surge en el XII Congreso Internacional de Geografía, celebrado en Cambridge en 1928. El autor de la propuesta fue Crawford, quien ante la Asamblea General del Congreso esbozó las principales características del proyecto. En una breve comunicación expuso que sería de gran utilidad desde un punto de vista histórico y geográfico la edición de las hojas del Mapa Internacional del Mundo a escala 1:1.000.000, correspondientes a la extensión del Imperio Romano, en las que se mostraran las principales vías de comunicación y otras características de su organización en el momento de su máxima extensión territorial. Para ello se utilizaría la base cartográfica de las hojas del Mapa Internacional a escala 1:1.000.000, proyecto que en ese momento se estaba elaborando en los distintos Institutos Geográficos europeos, que actuaban de forma homogénea coordinados por un Comité Internacional'.

En su primera andadura, el mapa no debía ser más que un mero esqueleto del mundo romano, que reflejara los asentamientos de nombre conocido y la red viaria principal. Adicionalmente se le podrían añadir otros datos como las zonas industriales (minas y alfares), topónimos (ríos, montes, bosques, pantanos) y etnónimos. En ciertas zonas, como Germania y Dacia, propuso introducir también las fronteras militares. En lo que se refiere a las fuentes antiguas sugirió utilizar preferentemente la información de los itinerarios y de los geógrafos grecolatinos (Ptolomeo, Itinerario de Antonino, Mapa de Peutinger, Notitia Dignitatum, etc). Era consciente de que un mapa de estas características necesitaba de la prospección y de la fotografía aérea como instrumentos esenciales de investigación, para comprobar sobre el terreno los datos conocidos mediante otros medios, por lo que propuso empezar por países en donde la investigación estaba más adelantada como por ejemplo la zona correspondiente a la hoja K-33, Roma, que abarcaba la mayor parte del centro y sur de Italia.

\footnotetext{
4. CRAWFORD, O.G.S., 1932, 1933, 1936, 1935 y 1938, 1937, 1950 y 1954/55.

5. PHILLIPS, C.W., 1959: 199 ss.

6. PIGGOTT, S., 1950: 185-6.

7. CRAWFORD, O.G.S, 1929: 48/ 230; 1929a: 414; 1939: 403-6.
} 
Crawford era consciente, y así lo planteó ante la Asamblea General del Congreso, de los principales problemas que iban a surgir. El primero, la colaboración internacional, dependía directamente de la aceptación que recibiese el proyecto en los diversos países. El segundo era el carácter marcadamente geográfico y cartográfico del proyecto. Para salvar la probable falta, en aquella época, de formación geográfica y cartográfica de los comités nacionales que se encargarían de la elaboración de la documentación histórica, solicitó al Comité Internacional del Mapa que se hiciera cargo de la dirección del proyecto y, por la misma razón, propuso que los directores de los Institutos Geográficos presidieran los distintos Comités Nacionales.

Su proyecto fue acogido por los participantes con gran entusiasmo y se nombró en ese momento una comisión, formada por cuatro miembros representantes de Gran Bretaña, Francia, Italia y España, para que se hiciese cargo del seguimiento del mismo ${ }^{8}$.

La comisión se reunió por primera vez el 30 de abril de 1929 en el Instituto Geográfico de Florencia; fue presidida por E.M. Jack, Director General del Ordnance Survey y contó con la representación de varias delegaciones ${ }^{9}$. En esta reunión se adoptaron las primeras decisiones sobre la forma y contenido del mapa, tomando como modelo la primera edición del mapa de Roman Britain de Crawford editado en 1924. Entre las decisiones adoptadas sólo nos referiremos a las más importantes, como las relativas a los sistemas de proyección y escala, que serían los mismos que los del Mapa del Mundo, 1: 1.000.000; número de hojas según la distribución del Mapa Internacional con la extensión que debía abarcar el proyecto (Fig. 1); la adjudicación de las hojas correspondientes a cada país (a España le correspondieron cinco (Fig. 2) y el establecimiento de la forma y tamaño de los signos convencionales. El período cronológico del mapa estaría comprendido entre la aparición de los primeros signos de la civilización romana y la caída del Imperio. Se acordó también que se hicieran tres ediciones simultáneas de cada hoja. En ellas debía rotularse en latín la toponimia más esencial. Llevarían, además de la inscripción latina, otras dos en cada edición: inglés y español en la primera, inglés y francés en la segunda y, en la tercera, inglés e italiano. Se anotarían todas las fechas conocidas con precisión, mientras que las probables se acompañarían de un signo de interrogación.

8. Presidente:E.M.Jack, Director General del Ordnace Survey.Vocales: Ch. de la Roncière, F. Pellati y Honorato de Castro. A propuesta del presidente, se nombró a Crawford secretario de la comisión. Véase, CRAWFORD, O.G.S., 1939: 48.

9. Inglaterra (O.G.S. Crawford), Italia (F. Pellati, G. Lugli, Thomas Ashby, buen conocedor de la topografía de Italia Romana, y el general N. Vacelli, Director del Instituto Geografico Militare de Florencia) y España (Honorato de Castro). El representante francés delegó en el presidente. Un primer informe de la reunión fue publicado por el Ordnance Survey a finales del mismo año, "The International Map of the Roman Empire (1:1.000.000), being the First Report of Comission No.7 of the International Geographical Union", Ordnance Survey Office (1912) 10 pp. y un mapa índice; Universo 12 (1931) p.675-9, que incluye un extracto a color de la K-33 (Roma). 


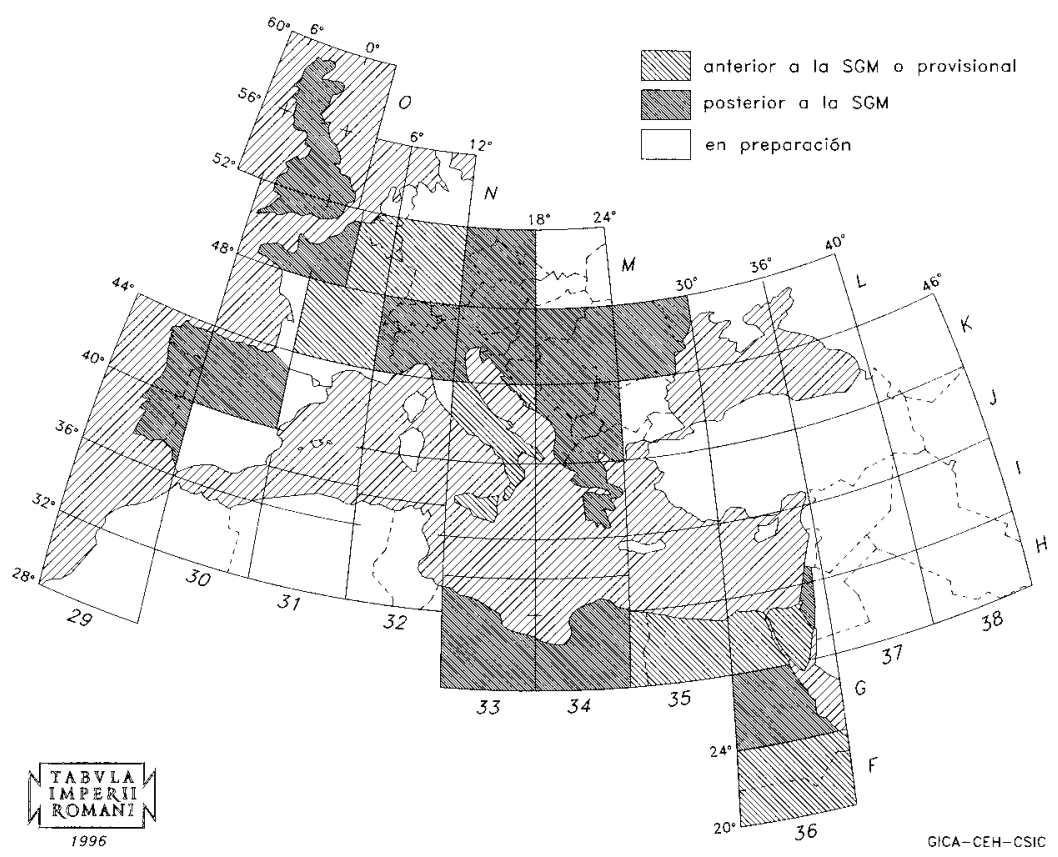

Fig. 1. Delimitación de las bojas correspondientes a España del proyecto TIR

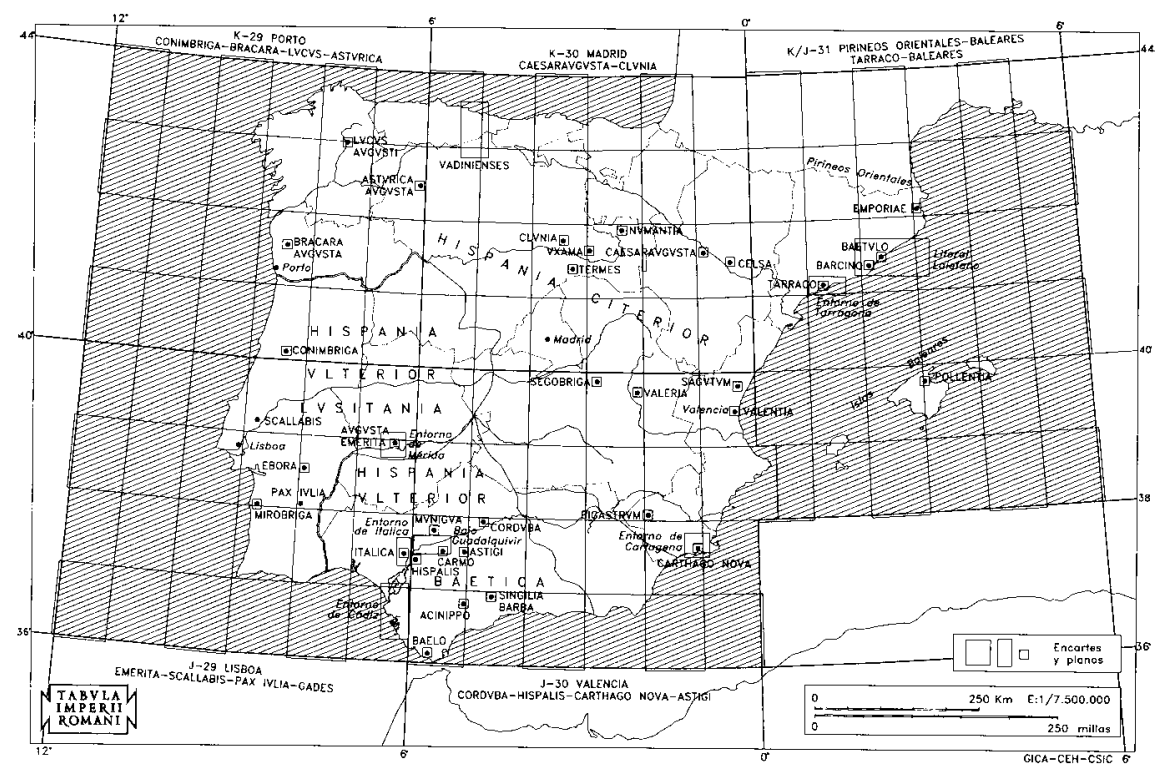

Fig. 2. Extensión del proyecto TIR con la delimitación de cada boja 
Junto a estas decisiones de caráter académico, se tomaron otras encaminadas a facilitar y agilizar lo más posible el funcionamiento del proyecto. El Comité Internacional solicitó la formación de Comités Nacionales formados por especialistas en trabajos cartográficos y en cuestiones arqueológicas e históricas. Se acordó proponer que cada Comité Nacional estuviera presidido por el Jefe del Servicio Nacional de Cartografía de cada país. El propio Comité Internacional hizo una propuesta de los nombres que podrían integrar los distintos Comités Nacionales ${ }^{10}$. Cada Comité Nacional aportaría todos los datos de carácter histórico-arqueológico que debieran figurar en el mapa y los entregaría al Servicio Nacional de Geografía para la elaboración de las minutas, que debían ser aprobadas por el Comité Internacional antes de su publicación. Se acordó aconsejar a los distintos Comités Nacionales el envío a los de otras naciones de cuantos antecedentes obraran en las bibliotecas que pudieran servir de material para la redacción del trabajo en las demás naciones. Finalmente, se aconsejó la publicación de todos los materiales utilizados, acompañando, a ser posible, gráficos y fotografías de la situación actual de los monumentos existentes.

La Comisión recalcó que el mapa habría de ser histórico, no arqueológico; es decir, no se pretendía elaborar una guía o índice de monumentos antiguos, sino mostrar de la mejor forma posible la distribución y características del poblamiento en época romana, toponimia latina de núcleos de población y accidentes geográficos ${ }^{11}$.

Las reuniones de la Comisión se suceden en París en $1931^{12}$, en donde se presentaron las pruebas de cuatro hojas: N-30, Edimburgo, K-33, Roma, K-29, Porto y K-30, Madrid (Figura 3); en Roma en 1932 $2^{13}$; en Varsovia en 1934 $4^{14}$,

10. Por lo que respecta a España, la Comisión propuso los siguientes miembros: PRESIDENTE: José de Elola y Gutiérrez, Director del Instituto Geográfico y Catastral.VOCALES: José Ramón Mélida Alinari, Catedrático de la Universidad Central y Académico de la Historia; Manuel Gómez Moreno y Martínez, Catedrático de la Universidad Central; Pedro Bosch y Gimpera, Catedrático de la Universidad de Barcelona;Antonio Blázquez,Académico de la Historia. Serían también vocales de este comité los designados por la Real Academia de la Historia y el Instituto Geográfico (Lorenzo Ortiz). Honorato de Castro, miembro de la Comisión Internacional, se incorporó a los trabajos y desempeñó en un principio la secretaría del Comité Nacional, aunque, poco después, ésta pasó a Lorenzo Ortiz. Antonio Blázquez renunció y le sustituyó Claudio Sánchez Albornoz.

11.Véase,ADAMS, F.W., 1954: vol.58, nº 1, p.58 y el manuscrito existente en el Instituto Geográfico Nacional gracias a D. José Cruz Almeida, Ingeniero Geógrafo, y que es probablemente un extracto de la reunión redactado por Honorato de Castro y fechado en Madrid a 7 de mayo de 1929.

12. Comptes Rendus du Congrès International de Géographie, Paris, 1931 (1934), vol.3, p.61728.

13. The International Map of the World 1:1.000.000: Report of a Conference beld in November 1932 at Rome in conection with the International 1/M Map of the Roman Empire. Central Bureau, Ordnace Survey Office, 1933, 8 pp., index map; Antiquity 7 (1933) 1-3.

14. Comptes Rendus du Congrès Internationale de Géographie, Varsovie, 1934 (1938), vol.4, p.73. Se presentan 6 hojas provisionales. Egipto (H-35, Alejandría, H-36, El Cairo, G-36, Aswan y F-36, Wadi Alfa), Francia (L-31, Lyon), Inglaterra (O-30, Aberdeen). 


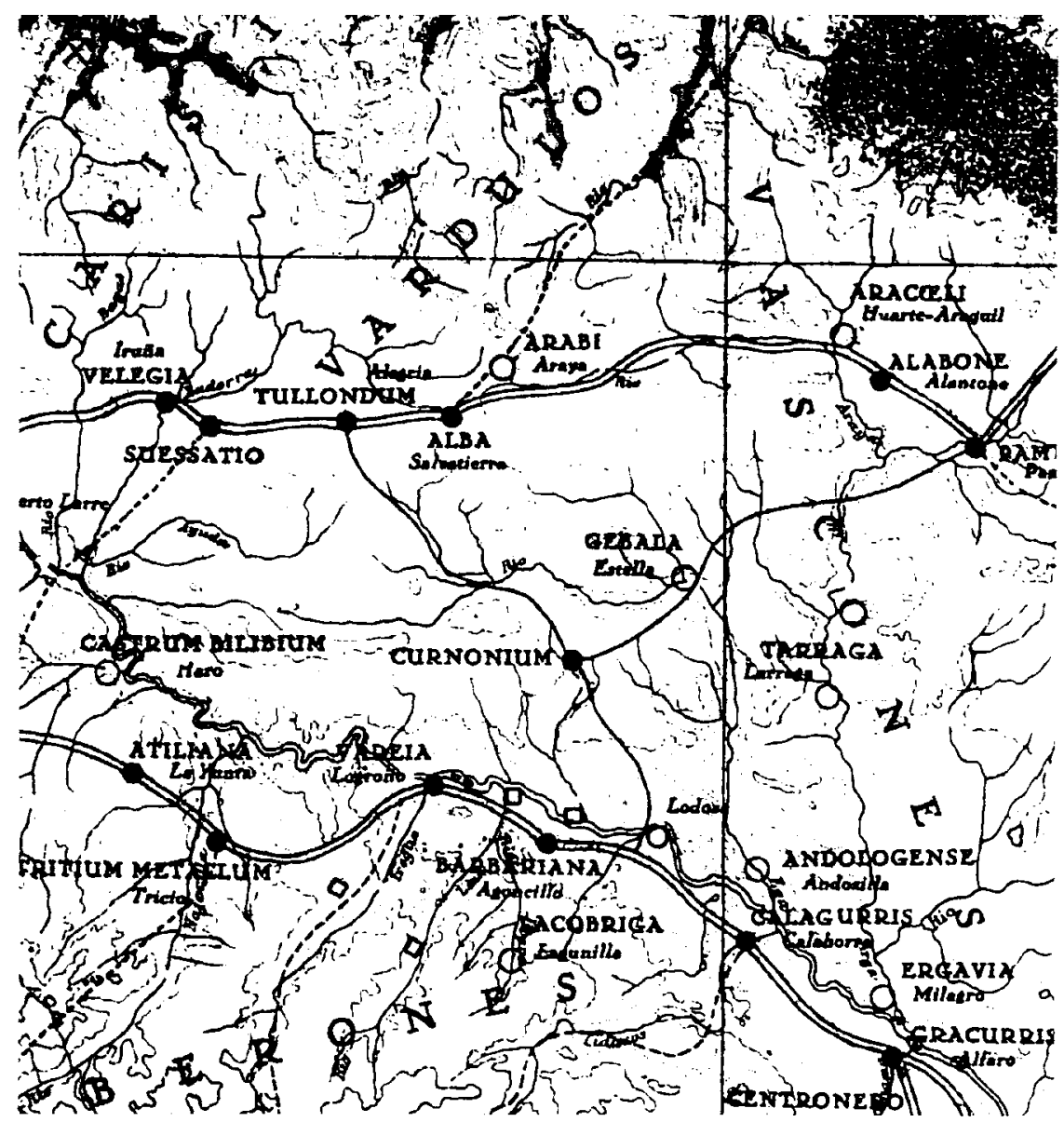

Fig. 3. Hoja, K-30, Madrid, 1931. Fragmento correspondiente a La Rioja

donde el proyecto fue oficialmente denominado Tabula Imperii Romani y, finalmente, en Londres en $1935^{15}$.

En 1935, el momento parecía excelente; se había conseguido una buena cooperación internacional y las perspectivas de futuro eran prometedoras. Crawford fue secretario del Comité Internacional desde 1928 a 1938 y durante este período salieron a la luz la mayor parte de las hojas, publicadas por ingleses que elaboraron tres cuartas partes de la zona que les correspondía: $\mathrm{N}$ -

15.Antes de la reunión, el Ordnance Survey publicó un folleto, Tabula Imperii Romani: Map of the Roman Empire based on the Carte International du Monde au Millionième, London Congress, 1935, Ordnace Survey Office, Southampton, 14 pp. and Index Map. El Congreso es comentado en The Geographical Journal 86 (1935) 523-526. 
30 (Edimburgo) en 1931 y O-30 (Aberdeen) en 1934.Además, en colaboración con el equipo francés y las autoridades gubernamentales egipcias, el Ordnance Survey editó en 1934 las hojas L-31 (Lyon), H-35 (Alejandría), H-36 (El Cairo), G-36 (Aswan) y F-36 (Wadi Halfa) ${ }^{16}$.Algunas hojas tuvieron en 1938 una segunda edición, como la de Lyon. Sin embargo, el proyecto no evolucionaba todo lo rápidamente que Crawford hubiera deseado. Las hojas italianas: L-32 (Milán), K32 (Florencia), K-33 (Roma) y J-33 (Palermo) y españolas: K-29 (Porto) y K-30 (Madrid), que estaban preparadas en 1931 y 1934, no se publicaron en este período.

Poco después estalló la Segunda Guerra Mundial. En 1940 los archivos del Ordnance Survey centralizados en Southampton fueron destruidos en un bombardeo.A partir de este momento, fue poco el tiempo y el dinero que se pudo utilizar para la continuación del proyecto. En 1938 Crawford dimitió como Secretario de la Oficina Central. Síntoma de su paralización es que no volvió a ser tratado en los siguientes Congresos Internacionales de Geografía ${ }^{17}$. En los años siguientes a la dimisión de Crawford sólo aparecieron dos hojas: la reedición de la hoja L-31 (Lyon) en 1938 y la edición de la hoja M-32 (Mainz) en 1940, que no se distribuiría hasta 1949 y que es la única de ese período que se ha podido manejar desde la Segunda Guerra Mundial ${ }^{18}$. Hay que esperar hasta 1954 para ver la publicación de nuevos trabajos, nuevamente elaborados por ingleses, que en 1954 publicaron la zona correspondiente a Libia: I/H-33 (Lepcis Magna) y I/H-34 (Cyrene) y, en 1958, la segunda edición de la hoja de Aswan (Coptos) ${ }^{19}$.

Al hacer la recensión de las hojas de Libia, Crawford expone claramente las razones por las que considera que el proyecto inicial había fracasado. Son las mismas que expuso en el XII Congreso Internacional de Geografía cuando se inició el proyecto: falta de colaboración internacional y, especialmente, ignorancia cartográfica de historiadores y arqueólogos que han hecho demasiado uso de la biblioteca y escaso trabajo de campo. Por lo que respecta a las hojas en donde más de un país estaba implicado, la principal causa estriba en la dificultad de obtener una colaboración internacional a nivel oficial, a la que se añaden los celos profesionales entre arqueólogos que parecen ser incapaces de colaborar conjuntamente. Sin embargo, el retraso afecta también a los países que tienen que trabajar aisladamente, como Italia. En este caso, Crawford seña-

16.A juzgar por el comentario de G. MacDonald en Journal of Roman Studies 25 (1935) p.24950 , las diferencias entre los trabajos, inevitables en todo proyecto que comienza, eran insignificantes.

17. Celebrados en Amsterdam (julio, 1938), en Lisboa (abril, 1949) y en Washington (agosto, 1952)

18. Revisada por M.P. Charlesworth, Antiquity 24 (1950) 147-8.

19. Revisada por D. Meredith, Geograpbical Journal 124 (1958) 554, para quien el mapa refleja una falta de conexión entre el cartógrafo y el arqueólogo, así como insuficiente trabajo de campo. 
la como motivos la total ignorancia cartográfica de los arqueológos, su apatía y la desidia de sus respectivos Institutos Geográficos. Confiesa que dimitió como secretario porque con muy pocas excepciones sus archaeological colleagues were a poor lot when it came to the compilation of a simple map ${ }^{20}$.

Su idea inicial era elaborar un mapa que reflejara los conocimientos del momento sobre el Imperio Romano en un período de tiempo razonable. Creía que el momento era adecuado, aunque quizá esperó demasiado de los Comités Nacionales o supuso que los servicios cartográficos europeos actuaban con la misma diligencia que el inglés. Su desilusión se produjo fundamentalmente por las hojas que empezaron a elaborarse a partir de 1929 y que todavía en 1945 no habían sido publicadas. La realidad es que el resto de Europa se movió a ritmos más lentos y la colaboración internacional tan pedida por Crawford llegó mucho más tarde y en momentos políticos más complejos.

En 1955 se publica la hoja de Praga, M-33 (revisada y ampliada en 1984). En 1961 aparece la hoja L-33 (Trieste). En 1965 la Academia Rumana publica un extracto de las hojas L-34, L-35, K-34 y K-35 correspondiente al territorio rumano. En 1966, se publica la hoja L-32 (Milán) ${ }^{21}$. En 1968 aparece la hoja L-34 (Budapest) con la colaboración de Yugoslavia, Rumanía, Bulgaria y Hungría. En 1969 se publica la hoja L-35 (Bucarest); en 1975 la hoja M-31 (París) y en 1976 la K-34 (Sofía). En 1987, se publica la tercera revisión de las hojas O y N-30 y se edita por primera la parte inglesa de la hoja N-31 (Amsterdam). Las últimas publicaciones corresponden a España (K-29, K-30 y J-29) e Israel (I-H, 36)

\section{ESPAÑA Y la TABULA IMPERII ROMANI}

Como se ha visto en páginas anteriores, España se incorporó al proyecto desde sus inicios y tomó parte muy activa en las primeras reuniones y en la elaboración de las primeras pruebas. En la historia de este proyecto se distinguen dos fases distintas separadas por la guerra civil. En un primer momento, el proyecto estuvo ligado a dos instituciones: la Real Sociedad Geográfica [RSG] e Instituto Geográfico Nacional [IGN] $]^{22}$. Posteriormente, cuando queda establecido que el proyecto dependa de los respectivos Institutos Geográficos Nacionales, el Instituto Geográfico Español se convirtió en el organismo a través del cual se canalizó la comunicación con el exterior y el lugar en donde se elaboraron los mapas.

20. CRAWFORD, O.G.S., 1954: 364-5.

21. Revisada junto con la hoja de Trieste por G.D. B. Jones, en The Geographical Journal 134 (1968) 452-3. La principal crítica es la incorrecta representación cartográfica, especialmente de las centuriaciones.

22. Boletín de la Real Sociedad Geográfica 68, 1928: 495-501; 69, 1929: 135. 

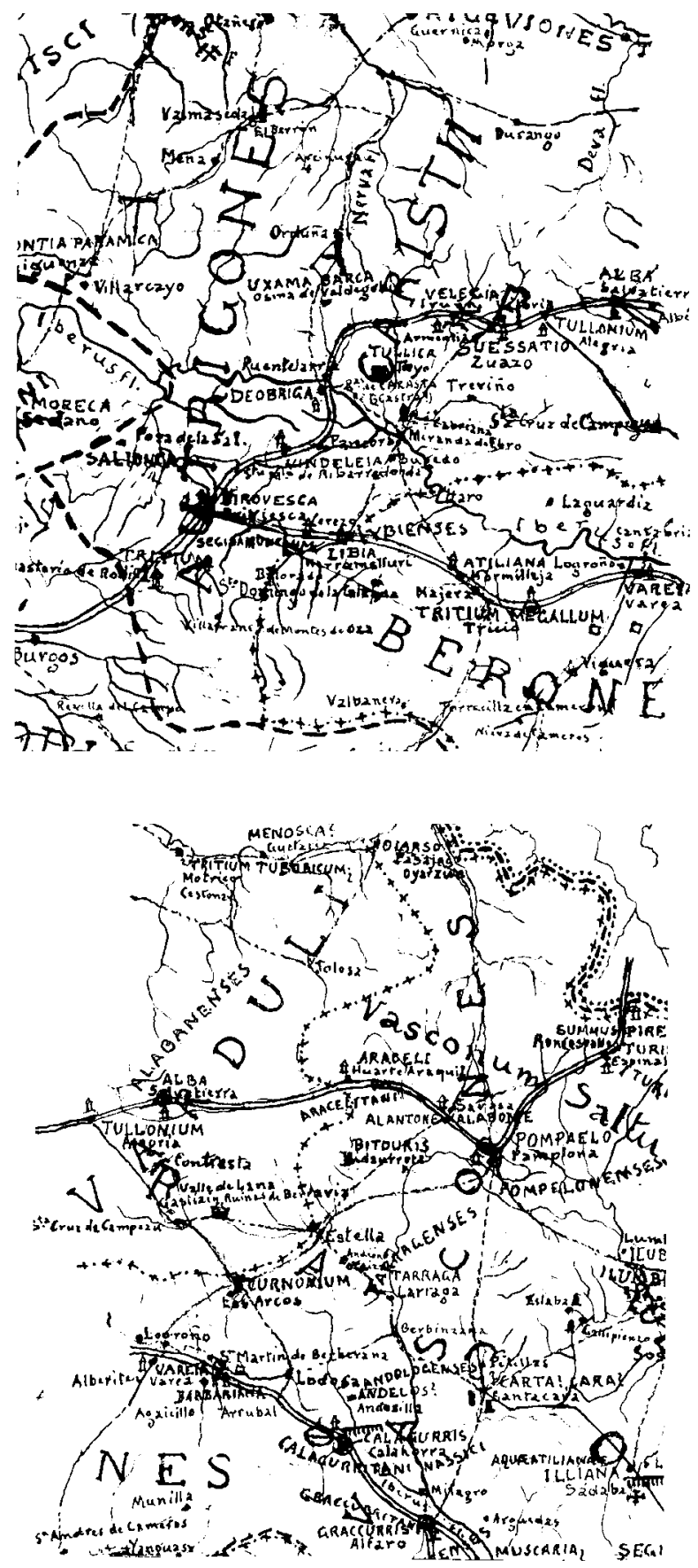

Figs. 4 y 5. Fragmentos del mapa de 1936 de La Rioja.. 
La documentación sobre la elaboración del mapa es muy escasa. Solamente en la actas de las sesiones de los miembros de la RSG reflejadas en su Boletín se pueden encontrar breves referencias sobre el nacimiento y gestación del proyecto. Paradójicamente, el Boletín de la Real Academia de la Historia no recoge datos sobre el mismo, a pesar de que los miembros del Comité Nacional eran académicos en su mayoría (Mélida, Sánchez-Albornoz, A. Blázquez y Gómez Moreno).

La única documentación accesible procede de una pequeña carpeta actualmente en el Instituto Geográfico Nacional que contiene breves notas sobre las primeras reuniones de Florencia, París y Roma, así como algunas cartas, que prueban la relación entre el Instituto Geográfico y la secretaría del Comité Internacional. Del proceso de elaboración de las dos primeras hojas no sabemos absolutamente nada. Hay que suponer que el Comité Español proporcionó al Instituto Geográfico la base documental histórico-arqueológica necesaria para la elaboración de los mapas. Lo cierto es que España presentó las hojas K29 (Porto) y K-30 (Madrid) en el Congreso de París ${ }^{23}$.

Durante los años 1934 y 1935 el proyecto no vuelve a ser tratado en las sesiones del Comité Nacional de la Unión Geográfica Internacional, pero tanto el Comité como el Instituto Geográfico siguieron trabajando. No sabemos la forma en que el proyecto fue financiado en el período de 1928 a 1931, pero, a partir de 1931, los gastos necesarios para cubrir las necesidades del Comité y de los colaboradores se cubrieron con partidas específicas del Presupuesto General del Estado. Como resultado de estos años de trabajo se conserva una minuta del mapa completo de la Península Ibérica firmada por Abelardo Merino, miembro de la Real Sociedad Geográfica, el 18 de mayo de 1936 (Figuras 4 y 5 ).

En la reunión de socios de la RSG celebrada el 18 de marzo de 1936, Abelardo Merino alude brevemente a los trabajos realizados en el mapa, del que proyecta publicar un extracto en el Boletín de la RSG, extracto que no aparecerá en los números siguientes. Esta es la última noticia que se tiene sobre el proyecto antes de la guerra civil ${ }^{24}$.

La documentación del período inmediatamente posterior a la guerra es muy escasa y procede de los fondos documentales del Departamento de Arqueología e Historia Antigua "Rodrigo Caro" del CSIC. Más allá de las disputas de carácter personal, a las que luego aludiremos, parece existir un rechazo de índole política, por lo menos hacia lo que significaban algunos de los miembros del comité anterior que habían participado en proyectos internacionales.

23. Boletín de la Real Sociedad Geográfica 76 (1936) p.239. Se encuentran varios ejemplares en la Cartoteca del Instituto Geográfico Nacional. R.A. Gardiner (1973, p.107) comenta que estas hojas nunca se publicaron debido al estallido de la guerra civil.

24. Boletín de la Real Sociedad Geográfica 76, 1936: 239. 
No olvidemos que Sánchez-Albornoz y Bosch Gimpera estaban en el exilio. Aunque el proyecto del Mapa del Mundo Romano siguió existiendo presupuestariamente, no parece que haya ningún tipo de conexión con el primitivo proyecto internacional, ya que su contenido y forma de elaboración difieren del proyecto anterior.

La primera noticia procede de una carta de Julio Martínez Santa-Olalla dirigida a Juan de Contreras, Marqués de Lozoya, entonces Director General de Bellas Artes y Director del Instituto de Arte y Arqueología “Diego Velázquez" del CSIC. En un tono altivo y autoritario le comunica su intención de ocuparse personalmente del tema (carta fechada el 19 de junio de 1940). Textualmente dice: "en vista del fracaso que con la presentación de una hoja del mapa del mundo romano de España obtuvo en una reunión del Comité Internacional celebrada en Roma y que ha servido para vergüenza de España, puesto que constantemente se presenta como modelo de lo que no puede ser un mapa arqueológico, trató el comité internacional de dicho mapa de buscar una solución, que permitiera el que dicha obra no se viera obstaculizada por la colaboración española" 25 .

A partir del año 1940 el proyecto se centraliza en el Instituto Diego Velázquez de Arte y Arqueología del CSIC, financiado por la Dirección de Bellas Artes del Ministerio de Educación Nacional, que libró sustanciosas cantidades al CSIC desde 1941 a 1945. Sin embargo, los proyectos de este Instituto se orientaban fundamentalmente por criterios tendentes a la exaltación de la monumentalidad y a la conservación del patrimonio nacional ${ }^{26}$.

En 1949 Bosch Gimpera escribe a Taracena comunicándole que en la última reunión de la Unión Académica Internacional, celebrada en Bruselas, se preguntó sobre si en España continuaba el trabajo que había emprendido hace años Claudio Sánchez-Albornoz y le propone que sea él mismo quien continúe el proyecto, añadiendo "que ha sido tema de conversación oficiosa la colaboración que el Consejo podría tomar en dicho proyecto". Le pide uno o dos ejemplares del mapa de España a escala 1:1.000.000 que necesita para los trabajos del mapa del mundo romano que la Unión Académica Internacional ha reemprendido (carta fechada el 3 de julio de 1949). Taracena contesta a Bosch

25. BALIL,A., 1984: 323; OLMOS, R. et al., 1993: 57 ss.

26. De hecho existen una serie de disposiciones legales que encomiendan al Instituto "Diego Velázquez" la labor expresa de catalogación o inventario de nuestro patrimonio arqueológico: Decreto 09.03.40 sobre el Catálogo Monumental de España, artículos 1 y 4; Decreto 19.04.41 sobre nuevas normas para el mismo Catálogo, artículo 3; Decreto 12.06.53 sobre Inventario del Tesoro Artístico Nacional, artículos 1, 26 (!se da un plazo de seis meses para su realización!) y 8. Sólo a partir de Decreto $\mathrm{n}^{\circ}$ 1938/1961 de 22.09.61 que crea el Servicio Nacional de Información Artística, Arqueológica y Etnológica, la labor que hasta entonces venía desarrollando el Instituto "Diego Velázquez" (y consecuentemente a partir de su creación, el Instituto "Rodrigo Caro") pasa a depender oficialmente de ese recien creado servicio. 
Gimpera en los siguiente términos: "respecto a la Forma Orbis Romani ya conoce Ud. el pasado. La consignación que para ello y bajo el título "Mapa Romano" venía figurando en presupuestos, pasó hace años y con el mismo epígrafe al Diego Velázquez y como la Forma Orbis Romani habrá quedado paralizada se ha venido aplicando a la carta arqueológica de la que también me ocupo personalmente en el Instituto". Por último, Serra Rafols acude a la Comisión de la $T I R$ celebrada en Paris en $1970^{27}$. Alude a una posible colaboración entre España y Francia para la K/J-31. Sin embargo, la elaboración de esta hoja no se llegó a realizar. Hay que esperar a 1986, fecha en que empieza lo que podríamos llamar la tercera, y última, fase del proyecto TIR-España, para que se inicie por fin la edición de las hojas de la $T I R$ correspondientes a la Península Ibérica.

Estado aCtual Del PROYeCto DE LA TIR EN EsPaÑa

A principios de la década de los 80, la Unión Académica Internacional ofreció al Prof.Antonio Balil, la posibilidad de volver a iniciar los trabajos de la TIR en España. Sin embargo, la falta de dotación económica del Centro de Estudios Históricos $(\mathrm{CEH})$ en ese momento paralizaron la continuación del proyecto. Sólo en 1985, a iniciativa del Dr. J. Arce, entonces Prof. de Investigación del Dpto. de Historia Antigua y Arqueología del CEH, se iniciaron los contactos entre la Vicepresidencia de Relaciones Internacionales del CSIC y el Prof. Carettoni,Vicepresidente del Comité Internacional de la $T I R$ y encargado de su seguimiento en las provincias occidentales del Imperio. Dichos contactos culminaron con la constitución del Comité Español de la $T I R$ y su inmediato reconocimiento por parte del Comité Internacional. Desde su constitución el Comité viene trabajando con una regular continuidad, presidido en la actualidad por el Prof. Domingo Plácido ${ }^{28}$.

27. Réunion de la Commission de la TIR (Paris, 26 enero 1970). Véase, Colloque International sur la Cartographie archéologique et bistorique, Paris, 1970 (Tours, 1972) p.239-241.

28. La dirección del Comité recayó en un primer momento en el Prof. Antonio Tovar; tras su muerte fue sustituido por el Prof. A. Balil, que a su vez fue sustituido por el Prof. G. Fatás. En la actualidad, está formado por los siguientes miembros: Presidente: Dr.D. Domingo Plácido (Univ.

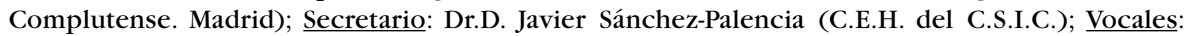
Dr.D. José María Alvarez (Museo Nacional de Arte Romano. Mérida), Dra.Dña. Carmen Aranegui (Univ. de Valencia), Dr.D. Javier Arce (Escuela de Arqueología. Roma); D. Ramón Corzo (Museo Casa Murillo. Sevilla), Dr.D. Juan Gil (Univ. de Sevilla), Dr.D.Antonino González (Univ. de Murcia), Dr.D. Josep Guitart (Univ. Autónoma. Barcelona), Dr.D. Gerardo Pereira (Univ. de Santiago de Compostela), Dr.D. Pedro Rodríguez (Univ. de Málaga), Dra. Dña.Adela Cepas (C.E.H. del C.S.I.), Dr.D. Luis Caballero (C.E.H. del C.S.I.C.), Dra. Dña. Carmen García (Univ. de Valladolid); Subdirector de Información e Investigación del $\mathrm{M}^{\mathrm{a}}$ de Cultura y Director de Procesos Cartográficos del IGN. 
Todos los miembros del comité pertenecen a organismos públicos de investigación situados en distintos puntos del territorio peninsular y en él están agrupados arqueólogos, historiadores y filólogos. El objetivo ha sido dar cabida en el proyecto a instituciones y organismos nacionales dedicados a la investigación de la España Romana y, al mismo tiempo, contar con la colaboración de arqueólogos especializados en las distintas zonas del país, para así poder acceder más fácilmente a la documentación disponible. En la primera reunión del Comité se marcaron las pautas de trabajo y se estableció la metodología. Nuevamente habría de realizarse en primer lugar la hoja K-29 (Porto) según las normas internacionales aceptadas para el proyecto $T I R$.

La estrategia del proyecto actual se basa en la recopilación de información que queda plasmada en fichas procesables. Unos equipos de apoyo, constituidos en los centros de investigación que trabajan habitualmente dentro del ámbito territorial de cada una de las cinco hojas y elaboran las fichas de información. Corre a cargo de estos equipos de apoyo la redacción inicial de las fichas técnicas correspondientes a su zona, la puesta al día de la información a partir de revistas y publicaciones de carácter local. La información de base es unificada, corregida y procesada informáticamente por un equipo de seguimiento y coordinación que actúa en el Dpto. de Arqueología e Historia Antigua del Centro de Estudios Históricos del C.S.I.C.

La informatización de la documentación básica se realiza mediante los programas DBase III Plus y Data Perfect.Ambos facilitan y agilizan el tratamiento y explotación de la documentación. Este proceso permite realizar tanto análisis temáticos de carácter general como estudios parciales por zonas. Mediante DBase III Plus se acumula un banco de datos con toda la información utilizada de fácil y rápido acceso que permite ordenar los datos tanto desde un punto de vista cronológico y geográfico (coordenadas, comunidad autónoma, provincia, municipio) como tipológico. Este banco de datos es susceptible de ser ampliado en cualquier momento, lo que permite realizar revisiones parciales o totales de cualquier zona del país. El segundo programa (Data Perfect) se utiliza para almacenar las fichas bibliográficas. Por otra parte, se ha llevado a cabo el tratamiento informático de las minutas cartográficas de cada una de las hojas, lo que supone incuestionables ventajas en la explotación de la documentación gráfica de los datos arqueológicos, mediante el programa CAD.

El resultado de este proceso queda reflejado en un mapa a escala 1:1.000.000 al que acompaña un índice topográfico donde se recogen tanto los diversos topónimos cartografiados como aquellos que no pueden ser localizados con precisión pero son mencionados por fuentes documentales. Como es lógico, la adecuación a la escala del mapa ha exigido unos criterios de valoración por parte del Comité de acuerdo con los usos de la TIR, que se resumen en los siguientes puntos: 
Los límites cronológicos abarcan desde la conquista romana hasta el siglo $\mathrm{V}$ d.C. La información más destacada la forman:

* Trazado de las vías principales: puentes y puertos de montaña, miliarios y mansiones. En el texto figura la toponimia moderna relacionada con las vías, allí donde se conservan con claridad restos de pavimentación o trazado, pero no se ha cartografiado para no sobrecargar el mapa.

* Ciudades importantes. Poblaciones.

* Asentamientos indígenas con ocupación romana.

* Villae. Sólo aquéllas de las quedan restos constructivos o se conoce la presencia de termas o de mosaicos.

* Monumentos aislados singulares o importantes, como santuarios y ninfeos, termas, acueductos, circos, arcos de triunfo o faros, presas y fuentes.

* Puertos marinos y fluviales, fondeaderos.

* Centuriaciones.

* Campamentos militares y campos de batalla.

* Instalaciones industriales: hornos de cerámica, alfares, factorías, minas, canteras y salinas.

* Ruinas y hallazgos aislados.

* Necrópolis aisladas. Se ha fijado como criterio general que deban contar con más de treinta enterramientos, pero también se ha valorado su importancia relativa dentro del contexto geográfico.

* Inscripciones. Sólo se incluyen las votivas dedicadas a deidades indígenas. De las funerarias, únicamente las concentraciones numéricamente significativas. Las conmemorativas aparecen sólo si hacen referencia al estatuto jurídico de poblaciones o miembros del ordo local.

* Accidentes geográficos y topónimos documentados por las fuentes literarias o epigráficas y de localización segura o probable.

Cuando ha sido necesario utilizar varios símbolos para cartografiar un determinado fenómeno, sólo figura el de carácter más amplio (ciudad o población, por ejemplo, y no teatro, necrópolis, inscripción, etc.). Sin embargo la descripción incluye los elementos no cartografiados. Los límites administrativos están trazados según la última bibliografía disponible.

Hasta el momento se han publicado ya tres hojas: hoja K-29, Porto (1991), que abarca el cuadrante NO de la Península Ibérica; K-30, Madrid (1993) (Figura 6), que abarca el Centro-Norte, y J-29, Lisboa (1995), que abarca el SE peninsular. La hoja K-31/J-31 (Pirineos Orientales-Baleares), que comprende $\mathrm{E}$ de Aragon, Cataluña, Baleares y N del País Valenciano, se editará en este año, quedando solamente la hoja J-30, Valencia, que probablemente saldrá a la luz a finales de este año o principios del próximo. 
La TIR no es exactamente una carta arqueológica. Tanto en España como fuera de ella, se han publicado numerosas cartas arqueológicas, siempre elaboradas a diferentes escalas y según distinta metodología. El interés de este proyecto radica en la visión de conjunto, inexistente por el momento, que puede proporcionar sobre toda la Hispania Romana, plasmando gráficamente el estado de la investigación y poniendo al alcance de los investigadores un verdadero diccionario topográfico. Su ejecución, coincidente en escala y criterios de definición

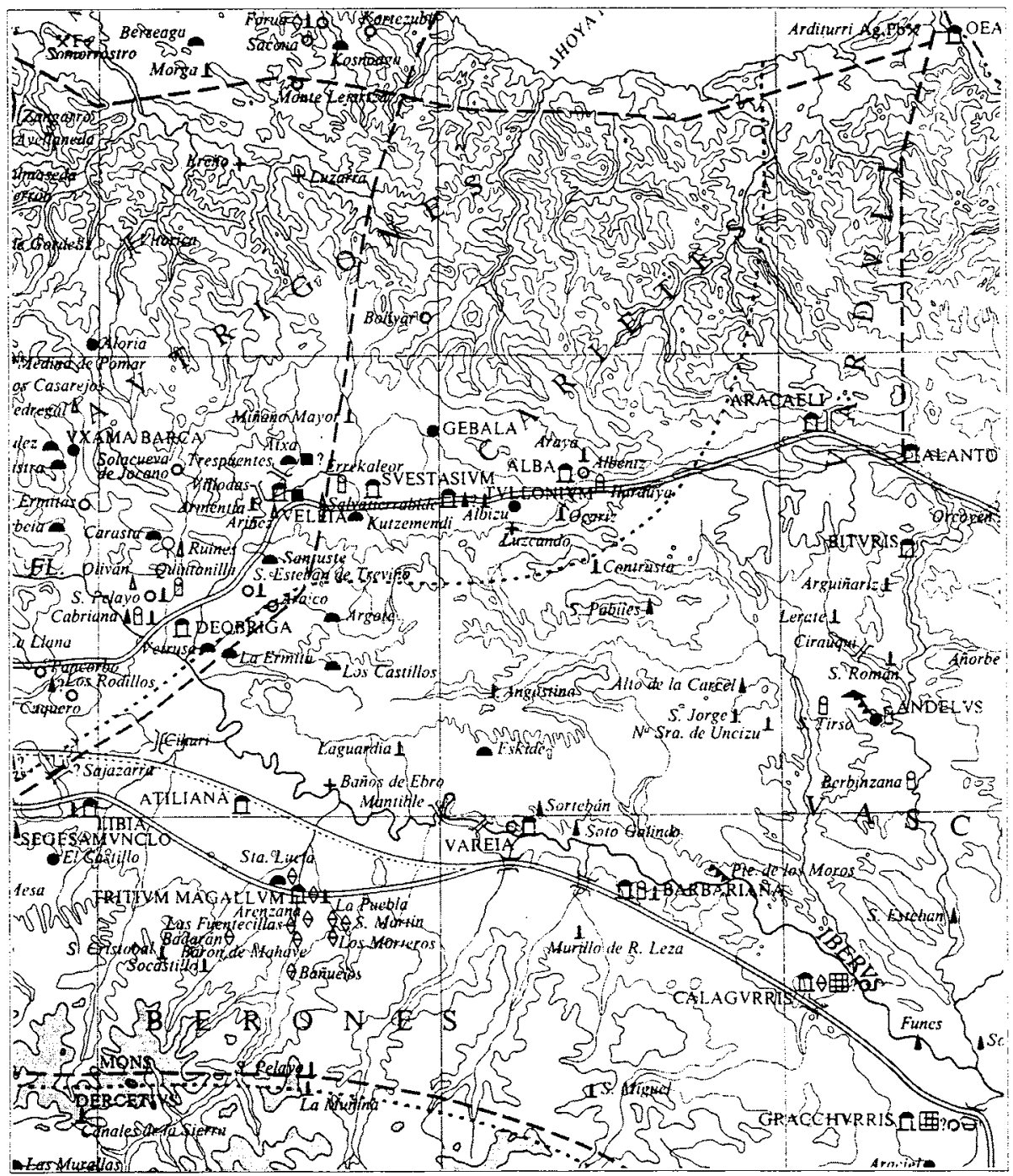

Fig. 6. Hoja, k-30, Madrid, 1993. Fragmento correspondiente a La Rioja. 
con el resto de las publicaciones internacionales de la $T I R$, permitirá abordar una planificación racional de las investigaciones sobre la Hispania Romana, además de otros posibles usos de carácter divulgativo o informativo, que podrán obtenerse de forma rápida gracias al banco de datos informatizado.

Para el buen desarrollo y finalización de este proyecto ha sido esencial el apoyo económico e institucional de organismos oficiales como el Ministerio de Cultura, el Consejo Superior de Investigaciones Científicas y el Instituto Geográfico Nacional, a quienes hay que agradecer su interés y colaboración. Sólo de este modo, con garantías de continuidad en un futuro, podremos salvar el considerable retraso de España con respecto al resto de los países comprometidos en el proyecto de la Tabula Imperii Romani, así como saldar la deuda contraída en 1932 por el Centro de Estudios Históricos con la Unión Académica Internacional. 


\section{BIBLIOGRAFÍA}

ADAMS, F.W., 1954

"Tabula Imperii Romani": AJA 58, n¹.

BALIL,A., 1984

"La Carta Arqueológica de España: precedentes, origen y desarrollo": Trabalbos de Antropologia e Etnologia, p.283.

CRAWFORD, O.G.S., 1929

Antiquity 3.

CRAWFORD, O.G.S., 1929a

Geographical Journal 74.

CRAWFORD, O.G.S., 1932

Map of Neolitic Wessex.

CRAWFORD, O.G.S., 1933

Map of Trent Bassin.

CRAWFORD, O.G.S., 1936

Map of South Wales.

CRAWFORD, O.G.S., 1935 y 1938

Map of Britain in Dark Ages.

CRAWFORD, O.G.S., 1937

Historic Maps of England and Wales.

CRAWFORD, O.G.S., 1939

"A proposed map of the Roman Empire": Report of the Proceedings of the International Geographical Congress, Cambridge, 1928.

CRAWFORD, O.G.S., 1949

The topography of Roman Scotland.

CRAWFORD, O.G.S., 1950 y 1954/55

Map of Monastic Britain.

CRAWFORD, O.G.S., 1953

Archaeology in the Field.

CRAWFORD, O.G.S., 1954

Geographical Journal 120.

GARDINER, R.A., 1973

"The International Map of the Roman Empire": The Geographical Journal 39.

KIRWAN, L.P., 1958

"Obituary, O.G.S. Crawford": The Geographical Journal 124. 
OLMOS, R. et al., 1993

"El origen de las cartas arqueológicas y el mapa del mundo romano": Inventarios y Cartas Arqueológicas. Homenaje a Blas Taracena (Soria, 1991), Valladolid.

PHILLIPS, C.W., 1959

"Archaeology and the Ordnace Survey": Antiquity 33.

PIGGOTT, S., 1950

"O.G.S. Crawford": Antiquity 50.

SECRETARIA DEL COMITE ESPAÑOL DE LA TABVLA IMPERII ROMANI 1990

"La Tabula Imperii Romani: su estado actual en España": AEA 63, 358-366.

TABVLA IMPERII ROMANII,

Drobeta-Romula-Sucidava. Bucarest, 1965.

TABVLA IMPERII ROMANII, L-32, Milano.

Mediolanum, Aventicum-Brigantium. Roma, 1966.

TABVLA IMPERII ROMANII, Hoja K-29:

Porto. Conimbriga, Bracara, Lucus, Asturica. Madrid, 1991.

TABVLA IMPERII ROMANI, Hoja K-30:

Madrid. Caesaraugusta, Clunia. Madrid, 1993.

TABVLA IMPERII ROMANII,

Iudaea - Palestina. Jerusalem, 1994.

TABVLA IMPERII ROMANII, Hoja J-29:

Lisboa. Emerita, Scallabis, Pax Iulia, Gades. Madrid, 1995.

TABVLA IMPERII ROMANII, Hoja K/J-31:

Pirineos Orientales, Baleares. Tarraco, Baliares. Madrid, 1996 (en prensa). 